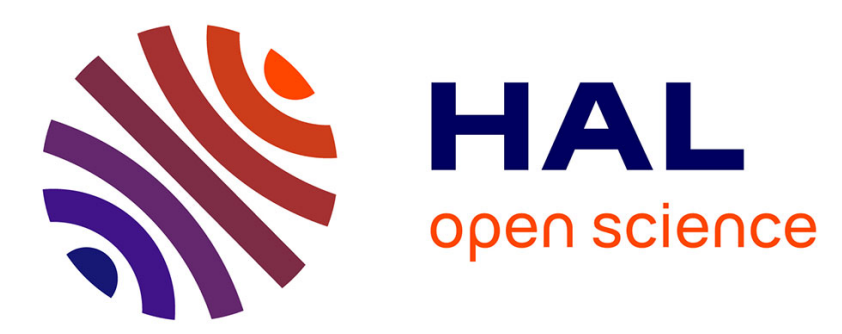

\title{
Linear elastic properties derivation from microstructures representative of transport parameters
}

\author{
M. T. Hoang, Guy Bonnet, H. T. Luu, Camille Perrot
}

\section{To cite this version:}

M. T. Hoang, Guy Bonnet, H. T. Luu, Camille Perrot. Linear elastic properties derivation from microstructures representative of transport parameters. Journal of the Acoustical Society of America, 2014, 135 (6), pp.3172-3184. 10.1121/1.4872296 . hal-01078343

\section{HAL Id: hal-01078343 \\ https://hal.science/hal-01078343}

Submitted on 10 Jun 2015

HAL is a multi-disciplinary open access archive for the deposit and dissemination of scientific research documents, whether they are published or not. The documents may come from teaching and research institutions in France or abroad, or from public or private research centers.
L'archive ouverte pluridisciplinaire $\mathbf{H A L}$, est destinée au dépôt et à la diffusion de documents scientifiques de niveau recherche, publiés ou non, émanant des établissements d'enseignement et de recherche français ou étrangers, des laboratoires publics ou privés. 


\title{
Linear elastic properties derivation from microstructures representative of transport parameters
}

\author{
Minh Tan Hoang, ${ }^{\text {a) }}$ Guy Bonnet, Hoang Tuan Luu, ${ }^{\text {b) }}$ and Camille Perrot ${ }^{\text {() }}$ \\ Université Paris-Est, Laboratoire Modélisation et Simulation Multi Echelle, MSME UMR 8208 CNRS, \\ 5 bd Descartes, 77454 Marne-la-Vallée, France
}

(Received 26 October 2012; revised 5 March 2014; accepted 7 April 2014)

\begin{abstract}
It is shown that three-dimensional periodic unit cells (3D PUC) representative of transport parameters involved in the description of long wavelength acoustic wave propagation and dissipation through real foam samples may also be used as a standpoint to estimate their macroscopic linear elastic properties. Application of the model yields quantitative agreement between numerical homogenization results, available literature data, and experiments. Key contributions of this work include recognizing the importance of membranes and properties of the base material for the physics of elasticity. The results of this paper demonstrate that a 3D PUC may be used to understand and predict not only the sound absorbing properties of porous materials but also their transmission loss, which is critical for sound insulation problems.
\end{abstract}

(C) 2014 Acoustical Society of America. [http://dx.doi.org/10.1121/1.4872296]

PACS number(s): 43.20.Jr, 43.35.Ns, 43.50.Gf [KVH]

Pages: $3172-3185$

\section{INTRODUCTION}

This paper presents a study that attempts to relate pore structure to both transport and elastic properties on the basis of easily measured single properties of porous materials. The authors are not aware of any comparable study, previous studies separating usually the prediction of transport and elastic properties. The porous materials under study are of the sort used extensively in the building and transportation industries for soundproofing applications. Previous works mainly focused on long-wavelength sound absorption through microstructured materials assumed to be non-deformable ${ }^{1}$ or on the effect of cellular morphology on the effective elastic properties of foam samples. ${ }^{2,3}$ Also, the published works show how the coefficients in poroelasticity equations can be calculated in principle from the microstructure, ${ }^{4-6}$ but often exclude applications to real samples except in a very limited number of cases: synthetic microstructures made of a regular arrangement of metallic hollow spheres ${ }^{7-9}$ and complex random geometries, such as a real sandstone reconstructed according to prescribed porosity and correlation length. ${ }^{10}$ Our opinion is that idealization of the porous medium through the reconstruction of a realistic three-dimensional periodic unit cell (3D PUC) is a key to understanding and predicting macroscopic behavior as it becomes possible to trace local geometry features from the microscale to the macroscale of day-to-day engineering applications. ${ }^{11,12}$ It was shown recently that threedimensional (3D) rigid-framed PUC can be used to capture the essential physics of the frequency-dependent fluidstructure interactions for a viscous and thermally conducting fluid saturating air-saturated foams. The rigid-frame porous

\footnotetext{
a) Also at: Faurecia Interior Systems, Acoustic TechCenter, Z. I. François Sommer BP 13, 08210 Mouzon, France.

${ }^{b}$ Also at: GAUS, Department of Mechanical Engineering, Université de Sherbrooke, Sherbrooke, QC J1K 2R1, Canada.

${ }^{c}$ Author to whom correspondence should be addressed. Electronic mail: camille.perrot@u-pem.fr
}

material assumption is still widely adopted to treat sound absorbing property applications in which the elastic properties of the skeleton do not play a significant role.

Although it has long been recognized that elastic foams are able to contribute to sound insulation, there is still no unified numerical homogenization approach which can make use of the cellular morphology to estimate both transport and elastic coefficients of a polyurethane foam sample with specified membrane content. The separate problems of the determination of transport properties on the one hand, and elastic properties on the other hand, knowing the microstructure, were considered extensively in the past. However, these problems are coupled through the microstructure properties and the present paper intends to show how this coupling allows providing data to be introduced when determining the elastic properties. Obviously, our approach has some similarities with the studies which aim at obtaining the linear elastic properties of solid foams by pore-scale simulation. This field of research has grown over the last decade and numerical simulations are mostly performed by finite elements and fast Fourier transform techniques. Gong et $a l^{2}$ compared the finite element approach and other models in order to predict the response of open cell foams to uniaxial compression by using the space filling Kelvin cell characterized by the geometric characteristics found in polyester urethane foams. ${ }^{2}$ The cells are elongated in the rising direction; the ligaments are assumed to be straight, and meant to have Plateau border cross-sections and nonuniform cross-sectional area distribution. The amount of material in the nodes is represented accurately. The linear elastic properties of the base material are measured directly from the foam ligaments. Comparison between measurements and predictions was satisfying. This paper also contains a thorough review of the elastic properties of solid foams to which the reader is referred. Sullivan et al. used finite element models of two elongated tetrakaidecahedron unit cells: one without cell faces ${ }^{13}$ and one with faces; $;{ }^{14}$ they illustrated it with analytical, numerical, and experimental data. Jang et al. built on the work of Gong et $a l^{2}$ by providing a more systematic and 

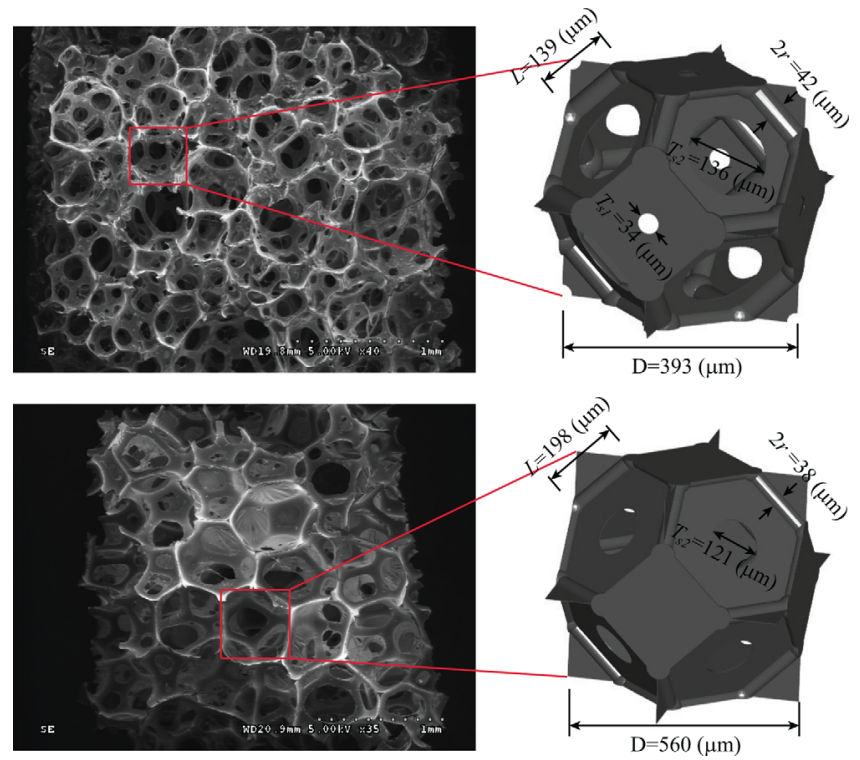

FIG. 1. (Color online) Geometrical characteristics of two different solid foam samples, $H_{1}$ (top) and $H_{2}$ (bottom).

detailed study of the microstructure of the same foams and using micro-computed $x$-ray tomography. ${ }^{3}$ The renderings are used in Finite Element (FE) models including irregular ones to estimate the elastic properties of the foams. The elastic moduli predicted by the Kelvin cell models were found to be within engineering accuracy of prediction for random foams. Thiyagasundaram et al. derived a finite element based micromechanics procedure to calculate the elastic properties of equisized and elongated tetrakaidecahedral unit cells without cell faces, which have a very high porosity and where the effect of varying strut cross-sections plays an important role. ${ }^{15}$

The purpose of the present work is to systematically study the effect of cellular morphology and membrane content on both transport and linear elastic properties of two real polyurethane foam samples, with and without solid films or membranes, with the solid geometry being the same as the one used for transport properties. The solid foam samples are chosen to cover different closure rates of membranes and elastic properties of the base material. The results of this work can then be used to relate the microstructural properties of a cellular foam structure and its closure rate of membranes to sound absorption and sound transmission loss of the poroelastic material. These results, together with a measure of the typical pore size through scanning electron micrographs, are used to reconstruct a 3D PUC with the corresponding closure rate of the membranes. Without any fitting parameters, the local characteristic sizes of the local geometry model, i.e., pore and throat sizes (see Fig. 1) are in close agreement with microscopy analysis (Fig. 2). This suggests that direct reconstruction of the 3D PUC could be done

A
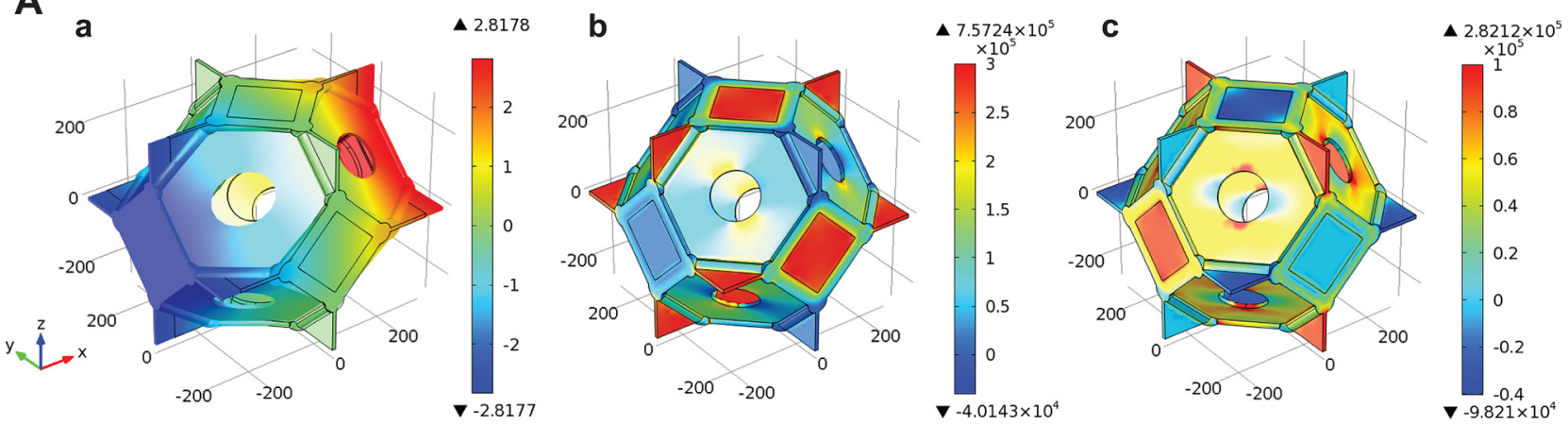

B
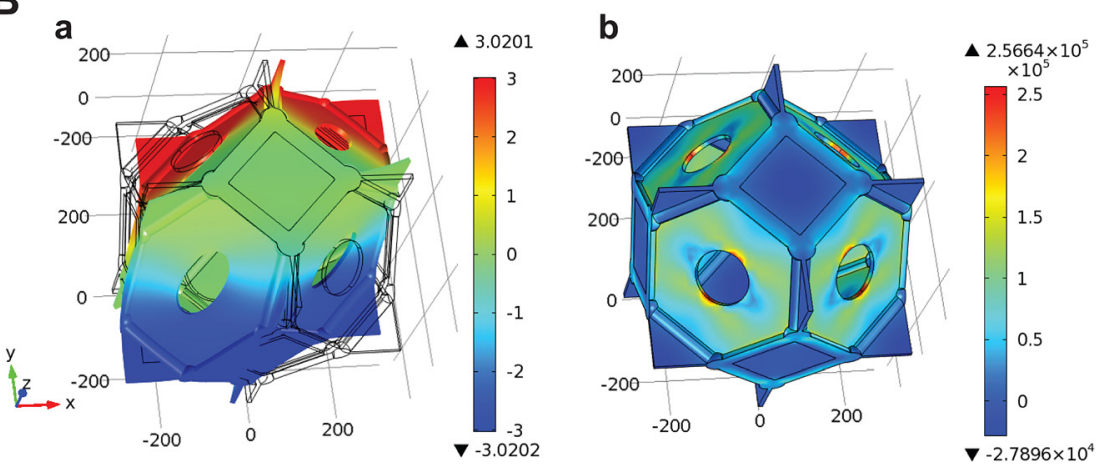

FIG. 2. (Color online) Numerical experiments allowing to identify the elastic constants $C_{11}, C_{22}$, and $C_{44}$ (in contracted notations) of the elasticity matrix for a solid foam model having cubic symmetry; illustrated with foam sample $H_{2}$. (A) Tensile strain numerical experiment. A uniform strain vector, $E=E_{11} \mathbf{e}_{1} \otimes \mathbf{e}_{1}$, is applied (along the principal coordinate direction [100]) in the equivalent homogeneous material with $E_{11}=0.01$ showing (a) the displacement field ( $\mu \mathrm{m}$ ), (b) the stress field $\sigma_{11}\left(\mathrm{~N} / \mathrm{m}^{2}\right)$, and (c) the stress field $\sigma_{22}\left(\mathrm{~N} / \mathrm{m}^{2}\right)$. The data are then averaged over the periodic unit cell as follows: $\Sigma_{11}=(1 / V) \int_{V} \sigma_{11} d V$, $\Sigma_{22}=(1 / V) \int_{V} \sigma_{22} d V$, and $\Sigma_{33}=(1 / V) \int_{V} \sigma_{33} d V$. Elastic constants, $C_{11}$ and $C_{12}$, are computed using the relations $\Sigma_{11}=C_{11} E_{11}$ and $\Sigma_{22}=C_{12} E_{11}$ (or $\Sigma_{33}=C_{12} E_{11}$ ). With a transversely isotropic configuration, the effective Young's modulus was found to be unchanged; $E_{T I}=E_{L}$ and $\nu_{T I}=\nu_{L}$. Contrary to the transversely isotropic configuration, the effective Young's modulus and Poisson's ratio for an isotropic material configuration were modified; $E_{I} \neq E_{L}$ and $\nu_{I} \neq \nu_{L}$. (B) Shear strain numerical experiment. A uniform strain vector, $E=E_{12}\left(\mathbf{e}_{1} \otimes \mathbf{e}_{2}+\mathbf{e}_{2} \otimes \mathbf{e}_{1}\right)$, is applied to the equivalent homogeneous material with $E_{12}=0.01$, showing (a) the displacement field $(\mu \mathrm{m})$ and (b) the stress field $\sigma_{12}\left(\mathrm{~N} / \mathrm{m}^{2}\right)$. The data are then averaged over the periodic unit cell as follows: $\Sigma_{12}=(1 / V) \int_{V} \sigma_{12} d V$ [Eq. (17)]. The last elastic constant, $C_{44}$, is computed using the relation $\Sigma_{12}=C_{44} E_{12}$. See also Sec. III. 
without any prior characterization of the static viscous permeability. Then, a micromechanical procedure is defined to compute linear elastic properties of the $3 \mathrm{D}$ reconstructed PUCs. It is proved that the linear elastic properties of the base material and the geometrical characteristics of membranes dominate the effective mechanical behavior of these poroelastic materials. This method accounts numerically for both visco-thermal dissipations and mechanical behavior caused by the presence of large membrane content in poroelastic foam submitted to long-wavelength acoustic waves.

The paper is organized as follows: Sec. II describes the reconstruction methodology, Sec. III presents the micromechanical analysis, and Sec. IV discusses the results in terms of the transport, mechanical, and acoustical quantities corresponding to microstructured poroelastic foams.

\section{MATERIALS AND METHODS}

\section{A. Regular solid foam structure with a specified closure rate of solid films or membranes}

The spatial structure of the solid foam models proposed here is derived from routinely available laboratory measurements (permeability, porosity, ligament length) and transport processes simulation inside porous media. A general view of the representation of solid foams and on the generation processes can be found in the studies of Perrot et al. ${ }^{16}$ and Hoang and Perrot. ${ }^{11}$ The solid foam models that are going to be used are spatially periodic in all directions of space. Hence, the unit cell provides the basic structure from which the whole medium can be derived by translation along three coordinate axes. Two different samples of solid foams were studied; we consider them more precisely in Fig. 1, which summarizes their more important geometrical characteristics. The ordered networks, analyzed in this article and shown in Fig. 1, are truncated octahedron networks with tetrahedral vertices; the ligaments of circular cross-section shape connect the spherical center of a regular tetrahedron, with plates either partially opened or not forming the faces of this polyhedron. This regular foam structure of cubic symmetry follows this geometry from an initially open-cell structure, whose close rate of solid films or membranes is allowed to grow. The cell size is provided by scanning electron microscopy (SEM) images and the closure rate of membranes is adjusted in order to comply with permeability data (see Ref. 11 for further details).

\section{B. Purely geometrical macroscopic properties}

Local geometry models corresponding to the real foam samples, namely, $H_{1}$ and $H_{2}$, were generated with the following measured porosity, ${ }^{17} \phi=0.93 \pm 0.01$ and $\phi=0.97 \pm 0.01$, respectively. In the first sample, the ligament length, $L$, is smaller with larger ligament thickness $2 r$ than in the second one (see Fig. 1 of this paper and Eq. (1) of Ref. 16).

The pore solid surface, $S$, and pore volume, $V_{p}$, were systematically calculated for each model of solid foam sample with elementary spatial integration. These two quantities can be combined in order to define the length scale,
$\Lambda^{\prime}=2 V_{p} / S$, a generalized hydraulic radius also named thermal characteristic length in the context of sound absorbing material. $^{18}$

\section{Transport parameters}

The macroscopic permeability, $k_{0}$, of each solid foam sample was measured. ${ }^{19}$ It was also computed on a unit cell considered as a representative volume element through solving the Stokes equations using a finite element method. ${ }^{11,16}$

The viscous characteristic length, $\Lambda$, can be used to characterize the throat size, $T_{S}$, of a porous medium. $\Lambda$ was introduced by Johnson et $\mathrm{al}^{20}{ }^{20}$ It is essentially a volume-tosurface pore ratio with a measure weighted by the local value of the velocity field, $\mathbf{v}_{\infty}(\mathrm{X})$, in a (non-viscous) potential flow,

$$
\Lambda=\frac{2 \int_{V p}\left|\mathbf{v}_{\infty}(\mathrm{X})\right|^{2} d V}{\int_{S}\left|\mathbf{v}_{\infty}(\mathrm{X})\right|^{2} d S}
$$

The tortuosity, $\alpha_{\infty}$, can be calculated when this velocity field is known

$$
\alpha_{\infty}=\frac{\left\langle\mathbf{v}_{\infty}(\mathrm{X})^{2}\right\rangle}{\left\langle\mathbf{v}_{\infty}(\mathrm{X})\right\rangle^{2}}
$$

where $\langle\cdot\rangle$ denotes a fluid phase average. This quantity was also obtained from our computation on the unit cell.

The static "thermal permeability" $k_{0}^{\prime}$ is related to the "trapping constant" $\Gamma$ of the frame by $k_{0}^{\prime}=1 / \Gamma .^{21}$ In the context of diffusion-controlled reactions, it was demonstrated by Rubinstein and Torquato ${ }^{22}$ that the trapping constant is related to the mean value of a "scaled concentration field," $u(\mathbf{r})$, by

$$
\Gamma=1 /\langle u\rangle
$$

where $u(\mathbf{r})$ solves

$$
\Delta u=-1 \text { in } V_{p}, \text { and } u=0 \text { on } S .
$$

The present study also reports computational results of $k_{0}^{\prime}$ for the considered cell structures. This parameter was shown to be very sensitive to membrane effects. ${ }^{11}$

\section{Effective mechanical properties}

The effective mechanical properties were obtained numerically for the two different solid foam models from finite element calculations and compared to values coming from experiments on real samples, namely, $H_{1}$ and $H_{2}$. The effective linear-elastic properties of the solid networks are determined by a finite element scheme operating on discretized representations of the structure; see Sec. III for further details. The material constituting the skeleton is assumed to be locally isotropic and linear-elastic; the elastic properties of the gaseous fluid phase are considered as negligible compared to the ones of the solid skeleton. 
The effective elastic longitudinal modulus, $E_{L}$, and Poisson's ratio, $\nu_{L}$, are obtained by applying two macroscopic external strains on the cube that bounds the solid foam model: a tensile strain and a shear strain related to the main coordinate directions. Since the solid foam models have cubic symmetry, three independent elastic constants, $C_{11}$, $C_{22}$, and $C_{44}$ (in contracted notations), exist in the elasticity matrix, whose identification requires two numerical experiments using periodic boundary conditions, to express $E_{L}$ and $\nu_{L}$. The effective elastic longitudinal modulus, $E_{L}$, and Poisson's ratio, $\nu_{L}$, are obtained by applying the $E_{L}=\left(C_{11}^{2}\right.$ $\left.+C_{11} C_{12}-2 C_{12}{ }^{2}\right) /\left(C_{11}+C_{12}\right)$ and $\nu_{L}=C_{12} /\left(C_{11}+C_{12}\right)$ formulas which are valid for the effective elastic properties along the principal coordinate directions, [100], [010], or [001] of materials with cubic symmetry.

The real porous frame does not have the cubic symmetry. On the contrary, real foams are either isotropic or transversely isotropic, the isotropy plane being orthogonal to the growing direction of the foam during the manufacturing process. However, the main idea is that "locally" the elastic properties of the material are correctly represented by a cellular model that exhibits cubic symmetry. The properties of "effective" materials having isotropic or transversely isotropic properties can be obtained by considering that the local cubic cells can have random orientations. Following this idea, the effective elastic properties of an effective transversely isotropic material (respectively, of an effective isotropic material) correspond to the properties obtained by random orientation of the unit cell when using arbitrary rotation around a given axis (respectively, when using any arbitrary rotation in space). It should be interesting to use the average of effective properties corresponding to different orientations of the unit cell. As is well known, this average corresponds to bounds on the effective properties. Thus, two bounds on the effective properties can be obtained, one given by averaging the elasticity tensor (components $C_{i j}$ ), the other by averaging the compliance tensor (components $S_{i j}$ ). Due to the fact that properties corresponding to different orientations display a moderate contrast, these two bounds are close and, in the following procedure, only the bound related to the average of compliances will be used. The detailed expression obtained by this angular averaging procedure yields, for the isotropic case, a lower bound for the Young's modulus of the isotropic material: $E_{I}=\left[5\left(C_{11}-C_{12}\right)\left(C_{11}+2 C_{12}\right) C_{44}\right] /\left[C_{44}\right.$ $\left.\left(3 C_{11}+C_{12}\right)+2\left(C_{11}-C_{12}\right)\left(C_{11}+2 C_{12}\right)\right]$, and an estimate of the Poisson's ratio $\nu_{I}=\left[-\left(C_{11}-3 C_{12}\right) C_{44}+\left(C_{11}-C_{12}\right)\right.$ $\left.\left(C_{11}+2 C_{12}\right)\right] /\left[C_{44}\left(3 C_{11}+C_{12}\right)+2\left(C_{11}-C_{12}\right)\left(C_{11}+2 C_{12}\right)\right]$.

Figure 2 shows the displacement and stress fields of the solid foam model corresponding to the real sample $\mathrm{H}_{2}$ during the shear strain and the tensile strain numerical experiments.

\section{MICROPOROELASTIC ANALYSIS}

\section{A. Elastic stiffnesses and compliances}

With respect to a fixed coordinate system, $\left(x_{1}, x_{2}, x_{3}\right)$, let $\sigma_{i j}$ and $\varepsilon_{i j}$ be the stresses and strains, respectively, in an anisotropic elastic material. The stress-strain law can be written as

$$
\sigma_{i j}=c_{i j k s} \varepsilon_{k s},
$$

in which $c_{i j k s}$ are the elastic stiffness coefficients that are components of a fourth rank tensor. They satisfy symmetry conditions. The inverse of this relation is written as

$$
\varepsilon_{i j}=s_{i j k s} \sigma_{k s}
$$

where $s_{i j k s}$ are the elastic compliances that are components of a fourth rank tensor. They possess the same symmetry conditions as the stiffness tensor.

\section{B. Contracted notations}

Introducing the contracted notation the stress-strain law (5) can be written as

$$
\sigma_{i}=c_{i j} \varepsilon_{j}, \quad c_{i j}=c_{j i} .
$$

In other words, due to the symmetry $\left(\sigma_{i j}=\sigma_{j i}\right.$ and $\varepsilon_{i j}=\varepsilon_{j i}$ ), only six independent components can appear in the stress and strain tensors. These six independent components of stress and strain can be "contracted" to a single index notation by writing

$$
\sigma_{i j}=\sigma_{k}, \quad \varepsilon_{i j}=\varepsilon_{k} ;
$$

and using for the substitution $(i, j) \rightarrow k$, the rule $(1,1) \rightarrow 1$, $(2,2) \rightarrow 2,(3,3) \rightarrow 3,(2,3)$ and $(3,2) \rightarrow 4,(1,3)$ and $(3,1) \rightarrow 5$, and $(1,2)$ and $(2,1) \rightarrow 6$. As a consequence, the fourth order elastic constant tensor may be contracted to a two-index notation by the application of the following conventions:

$$
\underset{\underline{\sigma}}{\underline{\sigma}} \underset{\sim}{\sigma}:=\left(\begin{array}{c}
\sigma_{11} \\
\sigma_{22} \\
\sigma_{33} \\
\sqrt{2} \sigma_{23} \\
\sqrt{2} \sigma_{31} \\
\sqrt{2} \sigma_{12}
\end{array}\right), \quad \text { and } \quad \underline{\underline{\varepsilon}} \rightarrow \underset{\sim}{\varepsilon}:=\left(\begin{array}{c}
\varepsilon_{11} \\
\varepsilon_{22} \\
\varepsilon_{33} \\
\sqrt{2} \varepsilon_{23} \\
\sqrt{2} \varepsilon_{31} \\
\sqrt{2} \varepsilon_{12}
\end{array}\right),
$$

the factor of $\sqrt{2}$ being inserted so that the equality $\underline{\sigma}: \underline{\varepsilon}=$ $\sigma \varepsilon$ holds true. Following this convention, the generalized Hooke's law relationship between the elements of the stress and strain tensor (represented as six element column vectors) can be compactly written in matrix notations as

$$
\underset{\sim}{\sigma}=\underset{\sim}{c} \underset{\sim}{\varepsilon}
$$

where $c$ is a six-by-six symmetric matrix. An expanded form of the $\tilde{\tilde{m}}$ atrix notation is given by

$$
\left(\begin{array}{l}
\sigma_{11} \\
\sigma_{22} \\
\sigma_{33} \\
\sqrt{2} \sigma_{23} \\
\sqrt{2} \sigma_{31} \\
\sqrt{2} \sigma_{12}
\end{array}\right)=\left(\begin{array}{cccccc}
c_{11} & c_{12} & c_{13} & c_{14} & c_{15} & c_{16} \\
& c_{22} & c_{23} & c_{24} & c_{25} & c_{26} \\
& & c_{33} & c_{34} & c_{35} & c_{36} \\
& \text { sym } & & c_{44} & c_{45} & c_{46} \\
& & & & c_{55} & c_{56} \\
& & & & & c_{66}
\end{array}\right)\left(\begin{array}{c}
\varepsilon_{11} \\
\varepsilon_{22} \\
\varepsilon_{33} \\
\sqrt{2} \varepsilon_{23} \\
\sqrt{2} \varepsilon_{31} \\
\sqrt{2} \varepsilon_{12}
\end{array}\right) .
$$


As indicated previously, it is frequently useful to express the strain in terms of the stress,

$$
\underset{\sim}{\varepsilon}=\underset{\sim}{s} \underset{\sim}{\sigma}
$$

where $\underset{\sim}{s}$ is the compliance tensor made of the 21 independent elements, $s_{i j}$. The quantity, $s$, is the inverse of $c$ in the matrix sense, $\underset{\tilde{z}}{s}={\underset{\tilde{z}}{c}}^{-1}$. The 21 coefficients, $s_{i j}$, are cẫlled the compliance constants.

\section{Material symmetries}

This paper addresses the linear elastic properties of partially closed cell solid foams with membrane-based tetrakaidecahedral cellular morphologies. This geometry exhibits a cubic symmetry. In this case, the elasticity tensor is defined by three independent coefficients, the elastic stiffnesses,

$$
C^{\text {cubic }}=\left(\begin{array}{cccccc}
C_{11} & C_{12} & C_{12} & 0 & 0 & 0 \\
& C_{11} & C_{12} & 0 & 0 & 0 \\
& & C_{11} & 0 & 0 & 0 \\
& & & C_{44} & 0 & 0 \\
& & & & & \\
& & & & & \\
& & & & & C_{44}
\end{array}\right) .
$$

Consequently, the elastic behavior can be described as based on only three independent elastic parameters, e.g., $C_{11}, C_{12}$, $C_{44}$. Alternatively, the elastic compliances, $S_{i j}$, might be expressed in terms of the elastic stiffnesses,

$$
\left\{\begin{array}{l}
S_{11}=S_{22}=S_{33}=\frac{C_{11}+C_{12}}{C_{11}^{2}+C_{11} C_{12}-2 C_{12}^{2}}, \\
S_{12}=S_{21}=S_{13}=S_{31}=S_{23}=S_{32}=\frac{-C_{12}}{C_{11}^{2}+C_{11} C_{12}-2 C_{12}^{2}}, \\
S_{44}=S_{55}=S_{66}=\frac{1}{C_{44}} .
\end{array}\right.
$$

Attention will be directed in the next sections to: (i) the specification of two kinds of numerical experiments required to completely characterize the elastic compliances and stiffnesses in terms of the base material's properties, and (ii) the obtention of averaged (a) transversely isotropic properties or (b) isotropic properties obtained from the results of the numerical experiments.

\section{Numerical experiments}

A simple tensile numerical experiment is such that $\sigma_{11}$ is different from zero and all other loads are null. Under these loading conditions, the relationship $\underset{\sim}{\varepsilon}=\underset{\sim}{S} \underset{\sim}{\sigma}$ greatly simplifies to yield

$$
\left\{\begin{array}{l}
\varepsilon_{11}=S_{11} \sigma_{11}, \\
\varepsilon_{22}=S_{12} \sigma_{11} \\
\varepsilon_{33}=S_{13} \sigma_{11} \\
\varepsilon_{23}=\varepsilon_{31}=\varepsilon_{12}=0 .
\end{array}\right.
$$

The longitudinal elastic modulus and Poisson ratios were deduced by definition

$$
\left\{\begin{array}{l}
E_{L}=\frac{\sigma_{11}}{\varepsilon_{11}}=\frac{1}{S_{11}} \\
\nu_{12}=\frac{\varepsilon_{22}}{\varepsilon_{11}}=-\frac{S_{12}}{S_{11}} \\
\nu_{13}=\frac{\varepsilon_{33}}{\varepsilon_{11}}=-\frac{S_{13}}{S_{11}}
\end{array}\right.
$$

For materials with cubic symmetry, substituting the expressions in Eq. (14) into Eq. (16) leads to

$$
\left\{\begin{array}{l}
E_{L}=\frac{C_{11}^{2}+C_{11} C_{12}-2 C_{12}^{2}}{C_{11}+C_{12}}, \\
\nu_{12}=\nu_{13}=\frac{C_{12}}{C_{11}+C_{12}},
\end{array}\right.
$$

which relates the longitudinal modulus and Poisson ratios to the elastic constants.

To calculate the macroscopic elastic constants of materials, a macroscopic strain is applied to the unit cell (Fig. 2). The displacement field inside the cell is the solution of the cell problem obtained from the homogenization of periodic media.

It is given by

$$
\mathbf{u}=\boldsymbol{E} \mathbf{x}+\mathbf{u}_{\text {per }},
$$

where $\mathbf{u}_{\text {per }}$ complies with periodicity conditions on the cell boundary. It can be shown ${ }^{23}$ that, accounting for the symmetries of the cell, these periodicity conditions can be changed into mixed boundary conditions enforcing that some components of $\mathbf{u}$ are equal to the similar components of $\boldsymbol{E} \mathbf{x}$, while expressing that the other components of the traction vector are null. For further details, the reader is referred to Sec. 4.2.1 of Ref. 24 from Michel et al. entitled "Symmetry conditions" and Appendix A of the same paper.

The components of the macroscopic effective stress tensor, $\sum_{\sim}$, induced by the macroscopic strain, $\boldsymbol{E}$, are obtained by averaging the local stress tensor, $\underset{\sim}{\sigma}$, obtained after solving the cell problem,

$$
\sum_{\sim}=\langle\underset{\sim}{\sigma}\rangle_{V}=\frac{1}{V} \int_{V} \sigma d V
$$

Yet, from another point of view, the microscopic stress tensor is related to the macroscopic strain tensor by

$$
\forall \underset{\sim}{E}, \quad \underset{\sim}{\Sigma}=\langle\underset{\sim}{\sigma}\rangle_{V}=\underset{\sim}{C} \underset{\sim}{E}
$$

This computation therefore produces some components of the elasticity tensor.

For the materials with cubic symmetry which contain only three independent elastic coefficients, only two numerical experiments are required to completely find out the elasticity matrix, one by using a macroscopic tensile strain and another one by using a macroscopic shear strain.

In a first step, we pay attention to a tensile strain numerical experiment for which we impose a uniform macroscopic 
strain, $\underline{\mathbf{E}}=E_{11} \mathbf{e}_{1} \otimes \mathbf{e}_{1}$, from which two elastic constants are found from the macroscopic stress tensor

$$
\left\{\begin{array}{l}
C_{11}=\Sigma_{11} / E_{11} \\
C_{12}=\Sigma_{22} / E_{11}
\end{array}\right.
$$

In a second step, we impose a uniform macroscopic strain, $\underline{\mathbf{E}}=E_{12}\left(\mathbf{e}_{1} \otimes \mathbf{e}_{2}+\mathbf{e}_{2} \otimes \mathbf{e}_{1}\right)$, to model a shear strain numerical experiment. This leads to

$$
C_{44}=\Sigma_{12} / E_{12}
$$

This completes the elasticity tensor.

\section{E. Macroscopically transversely isotropic and isotropic material configurations}

As explained before, the properties of "effective" isotropic and transversely isotropic materials can be obtained by a convenient angular averaging. The properties of an equivalent isotropic material can be built on the basis of the cubic cell by considering that the real material is made of cubic cells that have an arbitrary orientation. It can be obtained by computing the compliance tensor $S_{i j}^{\prime}(\psi, \theta, \varphi)$ for an arbitrary orientation of the axes of the cell, using the usual axis transformation of a tensor, and by averaging these properties over all orientations, i.e., on all possible Euler angles $(\psi, \theta, \varphi)$. As previously explained, this leads to an upper bound of the compliance tensor and a lower bound of the elasticity tensor,

$$
S_{i j}^{I}=\frac{1}{8 \pi^{2}} \int_{0}^{2 \pi} \int_{0}^{\pi} \int_{0}^{2 \pi} S_{i j}^{\prime}(\psi, \theta, \varphi) \sin \theta d \psi d \theta d \varphi .
$$

This finally produces a relationship between the lower bound of the components of the equivalent isotropic tensor, $S_{i j}^{I}$, and those of the elastic properties of the cubic material.

$$
\left\{\begin{array}{l}
S_{11}^{I}=S_{22}^{I}=S_{33}^{I}=\frac{3 S_{11}+2 S_{12}+2 S_{44}}{5} \\
S_{12}^{I}=S_{21}^{I}=S_{13}^{I}=S_{31}^{I}=S_{23}^{I}=S_{32}^{I}=\frac{S_{11}+4 S_{12}-S_{44}}{5} \\
S_{44}^{I}=S_{55}^{I}=S_{66}^{I}=\frac{2 S_{11}-2 S_{12}+3 S_{44}}{5}
\end{array}\right.
$$

Obviously, for the elastic material, these elastic constants are all functions of two elastic constants because $S_{66}^{I}+S_{12}^{I}=S_{11}^{I}$. For a transversely isotropic material, the computation is similar, but by restricting the random orientation of the axes to the ones perpendicular to the growth direction, i.e., by averaging over only all values of $\theta$

$$
S_{i j}^{T I}=\frac{2}{\pi} \int_{0}^{\pi / 2} S_{i j}^{\prime}(\theta) d \theta .
$$

This finally produces an upper bound of the components of the compliance tensor, which possess the properties of a transversely isotropic tensor, including $S_{22}^{T I}-S_{23}^{T I}=S_{44}^{T I}$

$$
\left\{\begin{array}{l}
S_{11}^{T I}=S_{11}, \\
S_{22}^{T I}=S_{33}^{T I}=\frac{3 S_{11}+S_{12}+S_{44}}{4} \\
S_{44}^{T I}=\frac{S_{11}-S_{12}+S_{44}}{2} \\
S_{55}^{T I}=S_{66}^{T I}=S_{44} \\
S_{12}^{T I}=S_{21}^{T I}=S_{13}^{T I}=S_{31}^{T I}=S_{12}, \\
S_{23}^{T I}=S_{32}^{T I}=\frac{3 S_{12}+S_{11}-S_{44}}{4} \\
S_{24}^{T I}=S_{42}^{T I}=0 \\
S_{34}^{T I}=S_{43}^{T I}=0 .
\end{array}\right.
$$

These elastic constants are only functions of five independent constants, as is well known for a transversely isotropic elastic material.

\section{RESULTS AND DISCUSSION}

\section{A. Geometrical and transport macroscopic properties}

Before looking at the mechanical results, let us consider the purely geometrical macroscopic properties $\left(\phi, \Lambda^{\prime}\right)$ and transport parameters $\left(k_{0}, \Lambda, \alpha_{\infty}\right)$, which were obtained on the cubic cell.

Note that in the regular solid foam structure, each unit cell can be generated by taking into account the experimental uncertainty in the input parameters $\phi \pm \Delta \phi$ $\left(\phi^{+}=\phi+\Delta \phi, \phi^{-}=\phi-\Delta \phi\right), k_{0} \pm \Delta k_{0}\left(k_{0}^{+}=k_{0}+\Delta k_{0}\right.$, $\left.k_{0}{ }^{-}=k_{0}-\Delta k_{0}\right)$, and $L \pm \Delta L\left(L^{+}=L+\Delta L, L^{-}=L-\Delta L\right)$. Such a generation process can be useful to obtain more detailed results for a given foam sample. For instance, the successive values of $\Lambda^{\prime}, \Lambda, \alpha_{\infty}$, and $k_{0}^{\prime}$ were computed using 1 (mean) $+2^{3}$ unit cells; and the standard deviation, $\Delta$, was subsequently calculated from these $1+2^{3}$ numerical results.

The porosity, $\phi$, was measured with a reasonable accuracy from the pressure/mass method. ${ }^{17}$ The permeability, $k_{0}$, was also directly measured as in Stinson and Daigle, ${ }^{19}$ after having removed a film on the surface of foam sample, $H_{1}$, whose presence is due to the injection process. Then, the determination of the missing parameters, $\Lambda^{\prime}, \Lambda, \alpha_{\infty}$, and $k_{0}^{\prime}$ is based on an inverse procedure ${ }^{25,26}$ using an analytical inversion from standing wave tube measurements ${ }^{27}$ and Johnson-Champoux-Allard-Lafarge model. ${ }^{18,20,21}$

The purely geometrical macroscopic properties $\left(\Lambda^{\prime}\right)$ and transport parameters $\left(\Lambda, \alpha_{\infty}, k_{0}^{\prime}\right)$ computed from the course of this multi-scale approach are in a rather good agreement with experimental data $\left(\Lambda^{\prime}, \Lambda, \alpha_{\infty}, k_{0}^{\prime}\right)$, especially when standard deviations are taken into account as seen in Table I.

Considering the experimental characterization as the ground truth, one might, however, find the coefficients $\Lambda$ to be slightly overestimated by the local geometry model, which occurs for idealized unit cells with monodisperse throat sizes [see Sec. IV B of Ref. 12, together with Eq. (8) of the same paper]. Indeed, the computed and measured values are-in the most favorable cases when taking the uncertainties into account — of similar magnitude for the $H_{1}$ foam sample [32\% of relative difference]; whereas, for the $\mathrm{H}_{2}$ foam sample a factor of at least 2 is observed [e.g., 
TABLE I. Macroscopic parameters: comparison between computational and experimental results. Experimental results include direct measurements of porosity $\phi$ (Ref. 17) and permeability $k_{0}$ (Ref. 19), and inverse characterization (Refs. 25 and 26) of the remaining parameters.

\begin{tabular}{|c|c|c|c|c|c|c|c|}
\hline Foam & Method & $\phi$ & $\Lambda^{\prime}(\mu \mathrm{m})$ & $k_{0}\left(\times 10^{-10} \mathrm{~m}^{2}\right)$ & $\Lambda(\mu \mathrm{m})$ & $\alpha_{\infty}$ & $k_{0}^{\prime}\left(\times 10^{-10} \mathrm{~m}^{2}\right)$ \\
\hline \multirow[t]{3}{*}{$H_{1}$} & Computation & & $146 \pm 22$ & & $55 \pm 6$ & $1.40 \pm 0.26$ & $28 \pm 12$ \\
\hline & Measurements & $0.93 \pm 0.01$ & & $5.35 \pm 0.42$ & & & \\
\hline & Characterization & & $143 \pm 57$ & & $33 \pm 4$ & $1.05 \pm 0.08$ & $55 \pm 28$ \\
\hline \multirow[t]{3}{*}{$\mathrm{H}_{2}$} & Computation & & $179 \pm 46$ & & $53 \pm 9$ & $2.40 \pm 0.55$ & $48 \pm 26$ \\
\hline & Measurements & $0.97 \pm 0.01$ & & $2.56 \pm 0.60$ & & & \\
\hline & Characterization & & $424 \pm 92$ & & $13 \pm 6$ & $1.58 \pm 0.64$ & $53 \pm 16$ \\
\hline
\end{tabular}

$(53-9) /(13+6)(231 \%$ of relative difference)]. Because the viscous characteristic length was determined for a periodic unit cell with a single closure rate of the membranes, the latter model is insensitive to the polydispersity of the throat sizes. More complex structures that contain, for example, additional throats of very small sizes could be used to extend this analysis. In agreement with this explanation, a microstructure analysis from SEM images is conducted in Sec. IV B, confirming that the overestimation of $\Lambda$ calculation was due to the fact that the model ignores the presence of many very small holes inside the membranes. While an excellent agreement is obtained between the experimental and numerical evaluations of the thermal characteristic length, $\Lambda^{\prime}$, for $H_{1}$, however, they differ significantly at first sight for $\mathrm{H}_{2}$. This discrepancy is due to the very low permeability of the latter foam sample, but also to the vibrations of its solid skeleton under acoustic excitation (see Secs. IV D and IVE for a detailed discussion of these coupled phenomena). The experimental estimation of $\Lambda^{\prime}$ in such circumstances is often affected by errors and uncertainties. This suggests that, in situations for which the classical experimental characterization methods are difficult to apply, a 3D PUC may be used as a tool which helps to interpret data, reduce uncertainties, and conclude on the validity of experimental results. Together with an overall relatively close match between the measured and computed macroscopic parameters $\left(\Lambda^{\prime}, \alpha_{\infty}, k_{0}^{\prime}\right)$, the former analysis about the origin of $\Lambda$ discrepancies and microstructural results below indicate that the local geometry models presented in Fig. 1 (with their local characteristic lengths summarized in Table II) capture the essential physics of the transports phenomena inside the foams.

\section{B. Analysis of the representativity of the microstructure from SEM imaging}

Note that in the case of high closure rate of membranes, as it happens for $\mathrm{H}_{2}$, the foam sample preparation may destroy some membranes, at least in the vicinity of the

TABLE II. Local characteristic length parameters of the microstructure models for foam samples, $H_{1}$ and $H_{2}$.

\begin{tabular}{lcccccc}
\hline \hline Foam & $L(\mu \mathrm{m})$ & $2 r(\mu \mathrm{m})$ & $D(\mu \mathrm{m})$ & $T_{s 2}(\mu \mathrm{m})$ & $T_{s 1}(\mu \mathrm{m})$ & $\delta / \delta_{\max }(一)$ \\
\hline$H_{1}$ & 139 & 42 & 393 & 136 & 34 & 0.65 \\
$H_{2}$ & 198 & 38 & 560 & 121 & 0 & 1.15 \\
\hline \hline
\end{tabular}

cutting tool. This tends to prevent an accurate quantitative characterization of the closure rate of membranes for real foam samples from micrographs. Nondestructive characterization techniques such as computed microtomography using $\mathrm{x}$-ray laboratory sources may also fail in this task due to their limited resolution. Keeping in mind these limits, it is, however, desirable to perform an investigation on the experimental closure rate of membranes measurable on SEM images. In order to reduce the projection bias, care was taken during measurements to select only ligaments and interconnections lying in the plane of observation (for three perpendicular surfaces). Interconnections were considered as ellipses on micrographs so that an estimate of their characteristic size, i.e., the throat size, $T$, was simply obtained by measuring and further averaging the two axes of inscribed ellipses. The cell sizes, $D$, were obtained from the measurement of ligament lengths, $L$, with $D=2 \sqrt{2} L$. The corresponding distributions are reported in Fig. 3. Unsurprisingly, the agreement between measured and computed cell size is good [Fig. 3(A)], because in this case ligament length measurements were taken as input values for the scaling of the local geometry model [see Fig. 2(b) of Ref. 11; note that a better agreement in Fig. 3(A)(b) of this paper simply requires a smaller closure increment $\Delta \delta$, like $\Delta \delta=\delta_{\max } / 30$ (where $\delta_{\max }$ was defined by $\left.\left.\delta_{\max }=L / 2-r\right)\right]$.

Looking now at the comparison between measured $\left(T_{m}\right)$ and computed $\left(T_{c}\right)$ throat sizes, one can see that the model is also consistent with real microstructure [Fig. 3(B)]. The local geometry model of foam sample $H_{1}$ displays two throat sizes, $T s_{1}$ and $T s_{2}$, with $T s_{1}=(L-2 r) \times\left(1-\delta / \delta_{\max }\right)$ and $T s_{2}=L \times\left(\sqrt{3}-\delta / \delta_{\max }\right)+2 r \times\left(\delta / \delta_{\max }-1\right)$. The associated values are $T s_{1}=34 \pm 15 \mu \mathrm{m}$ and $T s_{2}=136 \pm 15 \mu \mathrm{m}$. Because we also want to compare these throat sizes with a single scalar originating from measurements on SEM, we calculate $T_{c}$ by $T_{c}=\left(3 T s_{1}+8 T s_{2}\right) / 11$ - squared faces being shared by two cells. This yields $T_{c}=108 \pm 15 \mu \mathrm{m}$, a value fairly comparable to $T_{m}=92 \pm 57 \mu \mathrm{m}$ [Fig. 3(B)(a)]. Because the local geometry model of foam sample $\mathrm{H}_{2}$ exhibits only a single throat size due to a closure rate of membranes, $\delta / \delta \delta_{\max }>1$, this time $T s_{1}=0 \pm 7 \mu \mathrm{m}, T s_{2}=121$ $\pm 17 \mu \mathrm{m}$, and $T_{c}=121 \pm 22 \mu \mathrm{m}$ (for a single throat size, $T_{c}=T s_{2}$ ). This latter computed throat size is still in close agreement with the experimental one, $T_{m}=125 \pm 83 \mu \mathrm{m}$ [Fig. 3(B)(b)]. The larger relative proportion of small interconnections in the throat size distribution of foam sample $\mathrm{H}_{2}$ might explain why, in the single throat size model, its viscous characteristic length, $\Lambda$, is overestimated [Fig. 3(B)]. 

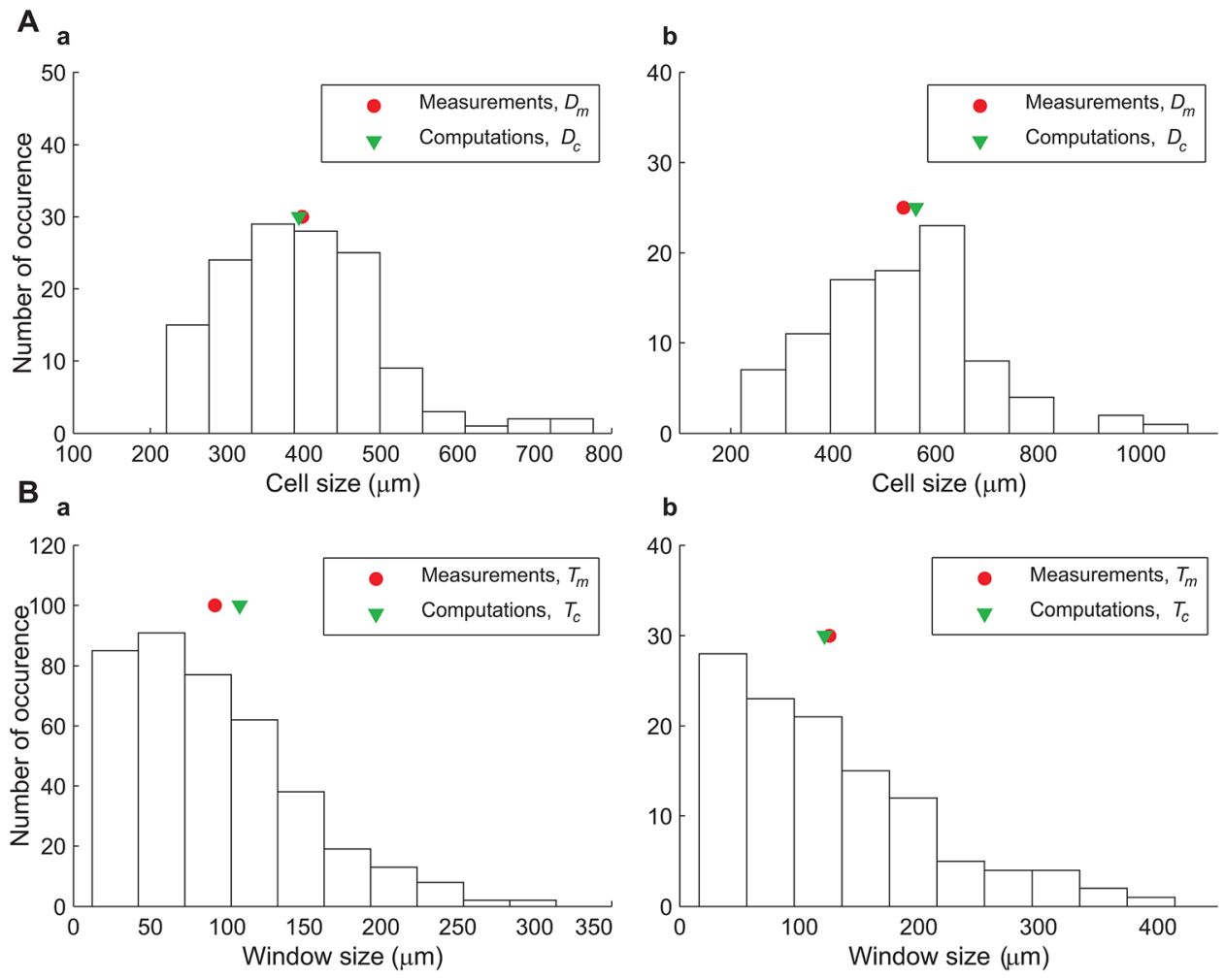

FIG. 3. (Color online) Distributions of (A) pore (top) and (B) interconnection or window (down) sizes for foam samples (a) $H_{1}$ (left) and (b) $H_{2}$ (right).

\section{Discussion on the relative influence of membrane closure rate and thickness}

The derivation of the local characteristic lengths of the model in the following subsection is based on a few assumptions related to the cellular morphology. This subsection aims at validating the effect of these assumptions on elastic properties with data coming from complementary experiments and modeling. Additional and more systematic quantitative comments can be given on the linear elastic properties of partially open cell foams to justify the use of the proposed models. Some results of the literature relative to the effects of cellular morphology modifications on the effective elastic properties are summarized below. We shall then compare them to the relative influence of membrane closure rate and thickness on the evolution of the effective Young's modulus.

\section{The effect of cross-section shape (at constant relative density or porosity)}

The Young's modulus is $38 \%$ higher for a given relative density if the ligament cross-sections are Plateau borders rather than equilateral triangles ${ }^{28}$ (analytical calculations).

\section{The effect of cross-sectional variation (at constant relative density or porosity)}

Foams with Plateau border cross-sections with uniform and nonuniform cross-sections were compared by Gong et $a .^{2}$ The effect of cross-sectional variation was found to be significant with the nonuniform cross-section foam being generally stiffer. For example, in the range of porosities (95\%-98\%), the nonuniform foam Young's modulus is $\sim 69 \%-74 \%$ higher than the uniform foam. This response is governed by the following adverse mechanisms. On the one hand, the Young's modulus to first order is governed by beam bending and, as a result, it is proportional to $\left(r_{0} / l\right)^{4}$, where $r_{0}$ is the radius of a three-cusp hypocycloid cross-section at mid-span of the ligament length, $l$. On the other hand, the nonuniformity makes the ligament stiffer by about a factor of 5 (difference between $C_{11} \simeq 0.017$ and $1 / 12$ ), which has a stronger effect than the difference in $r_{0} / l$. By contrast, the Poisson's ratio is only slightly reduced by cross-sectional nonuniformity.

\section{The effect of randomness (with a statistically identical foam morphology)}

Kelvin cell models and random foam models were compared by Jang et al. ${ }^{3}$ In all models, the cells were elongated in one direction and the ligaments were straight, but with a nonuniform cross-sectional area distribution. For nearly monodisperse foams, the random foams were found to be $5 \%-10 \%$ stiffer than the Kelvin cell models.

\section{The effect of correction for the volume of material in the nodes (at constant relative density or porosity)}

When ligaments are modeled as beams, each node connecting four of them, the ends of the beams overlap. The works of Gong et al. ${ }^{2}$ and Jang et al. ${ }^{3}$ account for excess material when calculating the material volume. For that purpose, they removed the excess material by cutting the ends of the beams with appropriately chosen smooth curved surfaces. Nodes were generated through that process for the Plateau borders, triangular, and circular ligament crosssections. Without this correction, the relative density is proportional to the respective geometric ratios raised to the power of 2, i.e., $\left\{\left(r_{0} / l\right)^{2},\left(a_{0} / l\right)^{2},\left(R_{0} / l\right)^{2}\right\}$ where $\left\{r_{0}, a_{0}, R_{0}\right\}$ are 
the characteristic cross-sectional dimensions at mid-span. With this correction, the power is no longer 2 and, furthermore, depends on the anisotropy according to a power-law relationship whose coefficients were fitted and listed by the authors. It might be noticed that, even for this advanced modeling work, some details of the real microstructure are omitted in the local geometry models such as some lumped material observed in closed faces. In this particular case, this results in estimated ligament dimensions somewhat larger than in the actual foams. In our work, the spherical nodes roughly account for the material concentration at the ligaments intersection. Let us mention that in actual measurements, uncertainty on porosity or relative density (taken as an input parameter for all of the aforementioned models), may have a stronger effect on ligament length estimates than accounting for excess material in the nodes when calculating the material volume.

\section{The effect of membrane thickness (at slightly varying relative density or porosity)}

The effect of membrane thickness on the elastic properties of foam samples $H_{1}$ and $H_{2}$ was found to be very significant with the thicker membrane being stiffer, and the stiffness being linearly dependent on the membrane thickness. For example, in the range of membrane thickness $(0-10 \mu \mathrm{m})$, the Young's modulus reaches an approximate value of $296 \%$ (respectively, 1244\%) higher than without membrane for foam sample $H_{1}$ (respectively, $H_{2}$ ). Clearly, the effect of membrane thickness is more significant for $\mathrm{H}_{2}$ than for $H_{1}$ because the closure rate of membranes $\delta / \delta_{\max }$ is higher for $H_{2}\left(\delta / \delta_{\max }=1.15\right)$ than for $H_{1}\left(\delta / \delta_{\max }=0.65\right)$.

\section{The effect of membranes' closure rate (at slightly varying relative density or porosity)}

The effect of membranes' closure rate on the elastic properties of foam samples $H_{1}$ and $H_{2}$ was also found to be very significant with higher closure rates corresponding obviously to stiffer materials. For example, in the range of membranes' closure rates $\delta / \delta_{\max }[0-2.043]$ and for a constant membrane thickness, the Young's modulus reaches an approximate value of $164 \%$ (respectively, $768 \%$ ) higher than without membranes for foam sample $H_{1}$ (respectively, $H_{2}$ ). Clearly, the effect of membranes' closure rate is more significant for $H_{2}$ than for $H_{1}$ because the porosity is higher for $H_{2}$ than for $H_{1}$.

Interestingly, the effects of membrane thickness and closure rate appear as having a stronger weight on the overall response of the foam sample linear elastic behavior compared to the effect of cross-section shape and cross-sectional variation of the ligaments (especially when porosity or relative density uncertainties are taken into account). This tends to justify the use of the proposed idealized unit cell. We shall now try to examine if these unit cells are also representative of independently measured effective linear poroelastic properties.

\section{Linear elastic properties}

The basic ingredients of flexible urethane foams of the type considered in this study are ester resin (or polyol), diisocyanate, water, catalysts, and surfactants. ${ }^{29,30}$ Foam $H_{1}$ was manufactured from the standpoint of these typical ingredients with a view of significantly lowering the Young's modulus of the resulting porous material when compared to standard plastic foams, and foam $\mathrm{H}_{2}$ is a commercial product. The samples represent cylindrical subsections of large panels of diameter equal to $44.5 \mathrm{~mm}$. Their heights are equal to $25 \mathrm{~mm}$ for $H_{1}$, and $10 \mathrm{~mm}, 15 \mathrm{~mm}$, and $20 \mathrm{~mm}$ for $H_{2}$.

Accurate literature values for the microscopic Young's modulus, $E_{\mu}$, and Poisson's ratio, $\nu_{\mu}$, are not available because these values are dependent on processing strategy. The values obtained in the literature for $E_{\mu}$ are scattered within a range of more than one order of magnitude; they are typically lying between 2 and $30 \mathrm{MPa} .{ }^{31} \mathrm{By}$ contrast, the microscopic Poisson's ratio seems relatively stable, with $\nu_{\mu}=0.25$. As mentioned by Gong et al., ${ }^{2}$ some foam chemists believe that the polymer flow resulting from the foaming process may cause preferential alignment of the long molecules of the material along the ligaments. Since these characteristics may not be easily achievable in bulk material, they recommend that the mechanical properties of the polymer be measured directly from foam ligaments. Therefore, the main conclusion which can be drawn is that specific measurements should be made on the material.

From the above analysis it can be concluded that because of the strong dependence of the foam properties on the base material, it is better to replace all results of the computations performed along the lines of Sec. III by non-dimensional values; for instance, $E_{I}^{n d}=E_{I} / E_{\mu}$. These non-dimensional data were first computed with membranes; a parameter which has a strong effect on the results is the membrane thickness (see Table III and Sec. IV C). An estimate of the membrane thickness $t=1.7 \pm 0.4 \mu \mathrm{m}$ was obtained by scanning electron micrographs. Two simulation results were given in Table III: with and without membranes.

The experimental values of macroscopic Young's modulus were obtained from compressional experiments and plotted in Fig. 4, which presents the evolution of the normalized Young's modulus, $E_{\exp }(\tau) / E_{\exp }^{\max }$, as a function of the degree of compression, $\tau$. They are obtained by uniaxial compression experiments ${ }^{32,33}$ on polyurethane foam samples and mainly correspond to the linear "compression of beam" regime (zone A) reported in the recent work of Geslain et al. ${ }^{34}$ (Sec. IV and references therein). The modulus during this regime can be approximated by a linear regression. $E_{\exp }^{\max }$ is the maximum value of $E_{\exp }(\tau)$ on the compression range of interest; $E_{\exp }^{\max }=E_{\exp }(\tau=0.035)=17.7 \mathrm{kPa}$ for $H_{1}$, and $E_{\exp }^{\max }=E_{\exp }(\tau=0.05)=122.7 \mathrm{kPa}$ for $H_{2}$. The slopes are, respectively, equal to 5.99 and 17.62 for foam samples $H_{1}$ and $H_{2} . H_{1}$ 's slope is smaller than $H_{2}$ 's slope, and clearly below the range of data presented by Geslain et al. (Table II of Ref. 34). This involves an unusually low sensitivity of the Young's Modulus with pre-constraint for foam sample $H_{1}$, which consequently acts as a guarantee to maintain a strong decoupling effect whatever the mounting conditions. This unusual property is consistent with special research efforts supporting the manufacturing process in collaboration with chemists. The asymptotic values of $E_{\exp }$ at $\tau=0$ correspond to the Young's modulus for a zero pre-constraint, yielding 
TABLE III. (a) Computed non-dimensional elastic properties of foam samples $H_{1}$ and $H_{2}$. In these simulations, the membrane thickness is equal to $10 \mu$ m. (b) Computed non-dimensional elastic properties of foam samples $\mathrm{H}_{1}$ and $\mathrm{H}_{2}$. In these simulations, the membrane thickness is equal to $1.7 \mu \mathrm{m}$.

\begin{tabular}{|c|c|c|c|c|c|c|c|c|c|c|c|}
\hline $\begin{array}{l}\text { (a) } \\
\text { Foam }\end{array}$ & Method & $\begin{array}{c}\sum_{11}^{n d} \\
\left(\times 10^{-3}\right)\end{array}$ & $\begin{array}{c}\sum_{22}^{n d} \\
\left(\times 10^{-3}\right)\end{array}$ & $\begin{array}{c}\sum_{12}^{n d} \\
\left(\times 10^{-3}\right)\end{array}$ & $\begin{array}{c}C_{11}^{n d} \\
\left(\times 10^{-3}\right)\end{array}$ & $\begin{array}{c}C_{12}^{n d} \\
\left(\times 10^{-3}\right)\end{array}$ & $\begin{array}{c}C_{44}^{n d} \\
\left(\times 10^{-3}\right)\end{array}$ & $\begin{array}{c}E_{T I}^{n d} \\
\left(\times 10^{-3}\right)\end{array}$ & $\begin{array}{l}\nu_{T I} \\
(-)\end{array}$ & $\begin{array}{c}E_{I}^{n d} \\
\left(\times 10^{-3}\right)\end{array}$ & $\begin{array}{l}\nu_{I} \\
(-)\end{array}$ \\
\hline \multirow[t]{2}{*}{$H_{1}$} & Without membranes & $0.13 \pm 0.02$ & $0.09 \pm 0.01$ & $0.03 \pm 0.01$ & $13.06 \pm 2.50$ & $9.33 \pm 1.36$ & $3.15 \pm 0.94$ & $5.28 \pm 1.57$ & $0.42 \pm 0.01$ & $4.79 \pm 1.40$ & $0.43 \pm 0.01$ \\
\hline & With membranes & $0.36 \pm 0.04$ & $0.14 \pm 0.01$ & $0.14 \pm 0.03$ & $36.01 \pm 3.72$ & $14.04 \pm 1.26$ & $14.44 \pm 2.71$ & $28.13 \pm 3.14$ & $0.28 \pm 0.01$ & $22.20 \pm 3.47$ & $0.32 \pm 0.01$ \\
\hline \multirow[t]{2}{*}{$\mathrm{H}_{2}$} & Without membranes & $0.04 \pm 0.02$ & $0.04 \pm 0.01$ & $0.00 \pm 0.00$ & $4.24 \pm 1.88$ & $3.72 \pm 1.45$ & $0.47 \pm 0.38$ & $0.76 \pm 0.62$ & $0.47 \pm 0.01$ & $0.71 \pm 0.57$ & $0.47 \pm 0.01$ \\
\hline & With membranes & $0.28 \pm 0.06$ & $0.12 \pm 0.02$ & $0.15 \pm 0.02$ & $28.43 \pm 6.01$ & $11.69 \pm 1.85$ & $15.02 \pm 2.39$ & $21.61 \pm 5.28$ & $0.29 \pm 0.01$ & 20.41 & $0.30 \pm 0.00$ \\
\hline (b) & & $\Sigma_{11}^{n d}$ & $\Sigma_{22}^{n d}$ & $\Sigma_{12}^{n d}$ & $C_{11}^{n d}$ & $C_{12}^{n d}$ & $C_{44}^{n d}$ & $E_{T I}^{n d}$ & $\nu_{T I}$ & $E_{I}^{n d}$ & $\nu_{I}$ \\
\hline Foam & Method & $\left(\times 10^{-3}\right)$ & $\left(\times 10^{-3}\right)$ & $\left(\times 10^{-3}\right)$ & $\left(\times 10^{-3}\right)$ & $\left(\times 10^{-3}\right)$ & $\left(\times 10^{-3}\right)$ & $\left(\times 10^{-3}\right)$ & $(-)$ & $\left(\times 10^{-3}\right)$ & $(-)$ \\
\hline \multirow[t]{2}{*}{$H_{1}$} & Without membranes & $0.13 \pm 0.02$ & $0.09 \pm 0.01$ & $0.03 \pm 0.01$ & $13.06 \pm 2.50$ & $9.33 \pm 1.36$ & $3.15 \pm 0.94$ & $5.28 \pm 1.57$ & $0.42 \pm 0.01$ & $4.79 \pm 1.40$ & $0.43 \pm 0.01$ \\
\hline & With membranes & $0.18 \pm 0.03$ & $0.10 \pm 0.01$ & $0.05 \pm 0.01$ & $17.62 \pm 2.57$ & $10.00 \pm 1.3$ & $5.34 \pm 1.07$ & $10.38 \pm 1.71$ & $0.36 \pm 0.01$ & $8.43 \pm 1.56$ & $0.38 \pm 0.01$ \\
\hline \multirow[t]{2}{*}{$\mathrm{H}_{2}$} & Without membranes & $0.04 \pm 0.02$ & $0.04 \pm 0.01$ & $0.00 \pm 0.00$ & $4.24 \pm 1.88$ & $3.72 \pm 1.45$ & $0.47 \pm 0.38$ & $0.76 \pm 0.62$ & $0.47 \pm 0.01$ & $0.71 \pm 0.57$ & $0.47 \pm 0.01$ \\
\hline & With membranes & $0.09 \pm 0.02$ & $0.05 \pm 0.01$ & $0.03 \pm 0.01$ & $8.77 \pm 2.33$ & $5.03 \pm 1.38$ & $3.27 \pm 0.68$ & $5.10 \pm 1.51$ & $0.36 \pm 0.02$ & $4.73 \pm 1.12$ & $0.37 \pm 0.01$ \\
\hline
\end{tabular}

$E_{\exp }(\tau=0)=14.4 \mathrm{kPa}\left(E_{\exp }(0) / E_{\exp }^{\max }=0.8138\right)$ for $H_{1}$ and $E_{\exp }(\tau=0)=5.4 \mathrm{kPa}\left(E_{\exp }(0) / E_{\exp }^{\max }=0.0441\right)$ for $H_{2}$. Note that the frequency-dependent characterization ${ }^{35,36}$ results were deliberately not presented in this paper because of the static nature of the proposed computational approach.

For comparison with the static computed results, the relative Young's moduli of both foams are given for ultimate values of $E_{\mu}(2-30 \mathrm{MPa})$; see Table IV. These values can be compared on the same table and through Fig. 5 with relative macroscopic Young's moduli coming from the computations.

Obviously, the range of the experimental relative moduli, $E_{\text {exp }} / E_{\mu}$, follows the range of the polyurethane base material modulus, $E_{\mu}$. However, some conclusions can still be drawn. It can be seen for $H_{1}$ that the estimation of the relative modulus with membranes is not far from the experimental one, providing that the Young's modulus of the base material is in the vicinity of the smallest values available in the literature (circle). For $\mathrm{H}_{2}$ foam sample, the value of the relative Young's modulus obtained with membranes is clearly within the admissible range of relative moduli, below

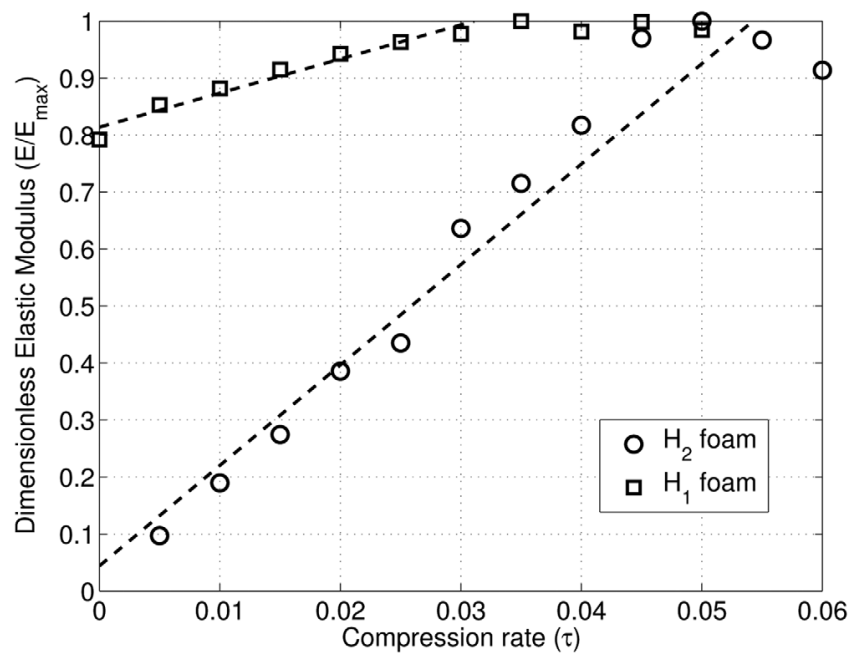

FIG. 4. The Young's modulus, E, measured from compression testing for foam samples, $H_{1}$ and $H_{2}$ as a function of the degree of compression $\tau$ (from the origin up to 0.06 ), and normalized by $E_{\max }$ [the maximum value of $E(\tau)$ on the compression range of interest]. the higher values, so that the Young's modulus of the base material must also be relatively low (square). From the above examples, it can be concluded that membranes must be modeled when the goal is to determine the homogenized linear elastic properties for foams containing a high closure rate of membranes. In the following, the experimental properties given in Table IV are used.

\section{E. Acoustical properties}

Let us go further to another possible consequence of this study, namely, the linear elastic properties derivation from microstructures representative of transport parameters and their effects on the acoustical characteristics of the poroelastic medium. The multi-scale determination of purely geometrical parameters, transport, and elastic properties was studied for the regular packing of partially open cell structures for two samples of solid foams. The numerical and experimental data relative to these macroscopic parameters are gathered in Tables I, III, and IV. These results can be used as input parameters of approximate but robust semiphenomenological models ${ }^{18,20,21}$ ("universal curves") as summarized in Appendix B of Ref. 16. We shall focus the presentation on the sound absorption, $\alpha(\omega)$, and sound transmission loss, $\operatorname{STL}(\omega)$, properties relative to the reconstructed poroelastic media (and we shall point out the features specific to each of these solid foams, $H_{1}$ and $H_{2}$ ). In what follows, measurement of the complex wave number and characteristic impedance of the foam samples was carried out using a three-microphone impedance tube setup as in the method introduced by Iwase $\mathrm{et} \mathrm{al.}{ }^{37}$ and further modified and discussed by Salissou and Panneton ${ }^{38}$ [see Fig. 1(a) of the latter reference]. The $\operatorname{STL}(\omega)$ estimates are modeled at normal incidence using transfer matrices ${ }^{39}$ for configurations with an impervious rigid backing at the rear face of the sample when assuming homogeneous, isotropic, and symmetric porous materials.

Some results relative to $\alpha(\omega)$ and $\operatorname{STL}(\omega)$ are displayed in Fig. 6. We first focus on the sound absorbing behavior. Real foam samples sound absorbing behavior is often believed to be widely governed by local visco-thermal dissipations that supposedly explain their effective properties. 
TABLE IV. Comparison between measured $E_{\text {exp }}$ and computed $E_{\text {comp }}$ elastic Young's moduli for a membrane thickness value $t=1.7 \mu$ m. $E_{\text {comp }}$ stands for the macroscopically isotropic computed value, $E_{I}$ [see Table III(b)]. $E_{\exp }=14.02 \mathrm{kPa}\left(\nu_{\exp }=0.16, \eta_{\exp }=0.29\right)$ and $E_{\exp }=11.93 \mathrm{kPa}\left(\nu_{\exp }=0.03, \eta_{\exp }=0.36\right)$ represent the measured Young's moduli (together with each corresponding Poisson's ratio and loss factor) for $H_{1}$ and $H_{2}$ foam samples at very small compression rates ( $\tau=0$ and $\tau=0.005$, respectively; see Fig. 4). Note that the extrapolated value at $\tau=0$ for $H_{2}$, deduced from linear least-squares regression of compressional measurements, is equal to $5.4 \mathrm{kPa}$.

\begin{tabular}{lcccc}
\hline \hline Foam & $\begin{array}{c}1000 E_{\text {exp }} \\
(\mathrm{Pa})\end{array}$ & $\begin{array}{c}1000 E_{\text {exp }} / E_{\mu(\min )} \\
(-)\end{array}$ & $\begin{array}{c}1000 E_{\text {exp }} / E_{\mu(\max )} \\
(-)\end{array}$ & $\begin{array}{c}1000 E_{\mathrm{comp}} / E_{\mu} \\
\text { (with membranes) }\end{array}$ \\
\hline$H_{1}$ & 14.02 & 7.01 & 0.46 & 8.43 \\
$H_{2}$ & 11.93 & 5.96 & 0.40 & 4.73 \\
\hline \hline
\end{tabular}

Yet, this assertion is, in general, correct, as we prove using semi-phenomenological models with a rigid or so-called "limp" frame assumption; see Fig. 6(A)(a) and Fig. 6(B)(a). Using our microscopic approach, we study the visco-thermal and structural dissipations in polyurethane foams and show that a rigid-skeleton assumption suffices to describe most of the sound absorbing behaviors of these poroelastic materials. A better agreement with experimental data might be obtained using a FE code for foam sample $\mathrm{H}_{2}$ by adjusting the Young's modulus to a value which corresponds to the state of the foam under compression when mounted in the tube. Here, our goal is to avoid using any fitting parameter to keep tracing local geometry features from the microscale to the macroscale. We note that by taking into account the experimental uncertainty in the input parameters, $\phi, k_{0}$, and $L$, the resonance falls within the error bars; see Fig. 6(B)(a).

The transmission of sound through such poroelastic matrials, on the contrary, cannot be predicted by a model in which the frame is motionless. In Fig. 6(A)(b), it is seen that a rigid model fails at describing the low frequency behavior of the sound transmission loss, whereas the acoustical behavior of foam sample $H_{1}$ of measured density equal to $68 \mathrm{~kg} / \mathrm{m}^{3}$ is well described by a limp model; ${ }^{40}$ see Fig. 6(A). It is possible to verify the pertinence of the limp model from the experimental results measured with a standing wave tube, since $\lim _{\omega \rightarrow 0} \operatorname{STL}(\omega)=0$ (rigid body motion of the

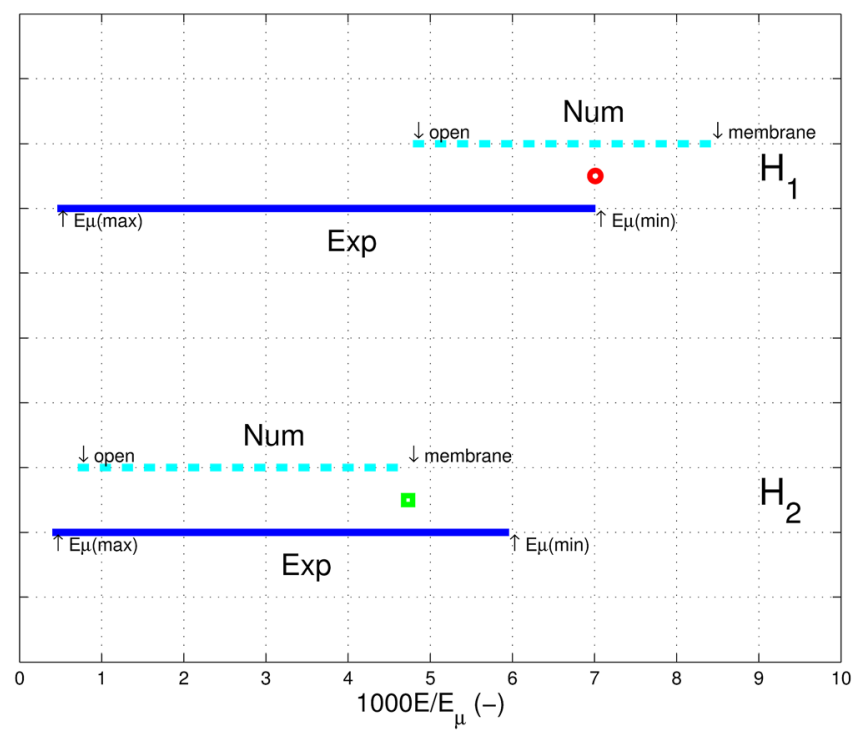

FIG. 5. (Color online) Comparison between non-dimensional experimental and numerical quasi-static Young's moduli for $H_{1}$ (top) and $H_{2}$ (bottom) foam samples. frame); see Fig. 6(A)(b). Using the measured density of $\mathrm{H}_{2}$ of $25 \mathrm{~kg} / \mathrm{m}^{3}$, the sound absorbing behavior of the low-density foam was estimated using a limp model in a good agreement with the impedance tube measurements; see Fig. 6(B)(a). The additional sound absorption generated using a limp model for the low density foam was determined to be due to an added rigid body motion of the overall sample (because of the apparent mass added in the equivalent fluid at the resonant frequency). The sound transmission loss that dropped in the low frequency range with the resonance frequency was more sensitive to elastic properties compared with sound absorbing properties whose essential behavior can be captured with a limp model.

Using the elastic properties of the foam sample $\left(H_{1}\right)$, which can be established using the method of micromechanics described in this paper, we obtain a prediction for the sound absorption and sound transmission loss in agreement with experimental results in a standing wave tube; see Fig. 6(A). This is done without prior experimental knowledge of the sound transmission loss behavior in the asymptotic low frequency range. Figure 6(B) also illustrates the utility of the elastic results derived in the preceding sections. We begin with considering the frame acoustical excitability (FAE) of the foam sample. ${ }^{41}$ By computing the FAE, our interest is (i) to examine the edge constraint effect on the acoustic behavior of the foam and (ii) to illustrate the effect of the elastic properties of the foam on the behavior of $\alpha(\omega)$ and $\operatorname{STL}(\omega)$ in agreement with the results simulated in a standing wave tube numerical experiment. The values of permeability, $k_{0}$, and bulk density, $\rho_{1}$, are much lower for $H_{2}$ $\left(\rho_{1}=25 \mathrm{~kg} / \mathrm{m}^{3}\right)$ than for $H_{1}\left(\rho_{1}=68 \mathrm{~kg} / \mathrm{m}^{3}\right)$. For foam samples $H_{1}$ and $H_{2}$ having identical thicknesses and radii with a similar stiffness, we note that the value of the FAE for $\mathrm{H}_{2}$ increases over more than 1 order of magnitude. This indicates that the boundary conditions in the tube will influence the overall sound absorption and sound transmission results, and their effect must be simulated within an axisymmetric poroelastic FE formulation of the Biot-Allard equations ${ }^{42-45}$ for better agreement with the experiments. Details on the axisymmetric FE poroelastic formulation can be found in Refs. 46 and 47. As previously discussed, our estimates using the elastic properties of the frame with a Biot-Allard model are close to laboratory measurements of sound absorption; see Fig. 6(B)(a). By contrast, it was also necessary to use an axisymmetric poroelastic FE formulation to properly model the bounded edge boundary condition for the sound transmission loss because the frame acoustical excitability was quite strong; see Fig. 6(B)(b). Meanwhile, we 

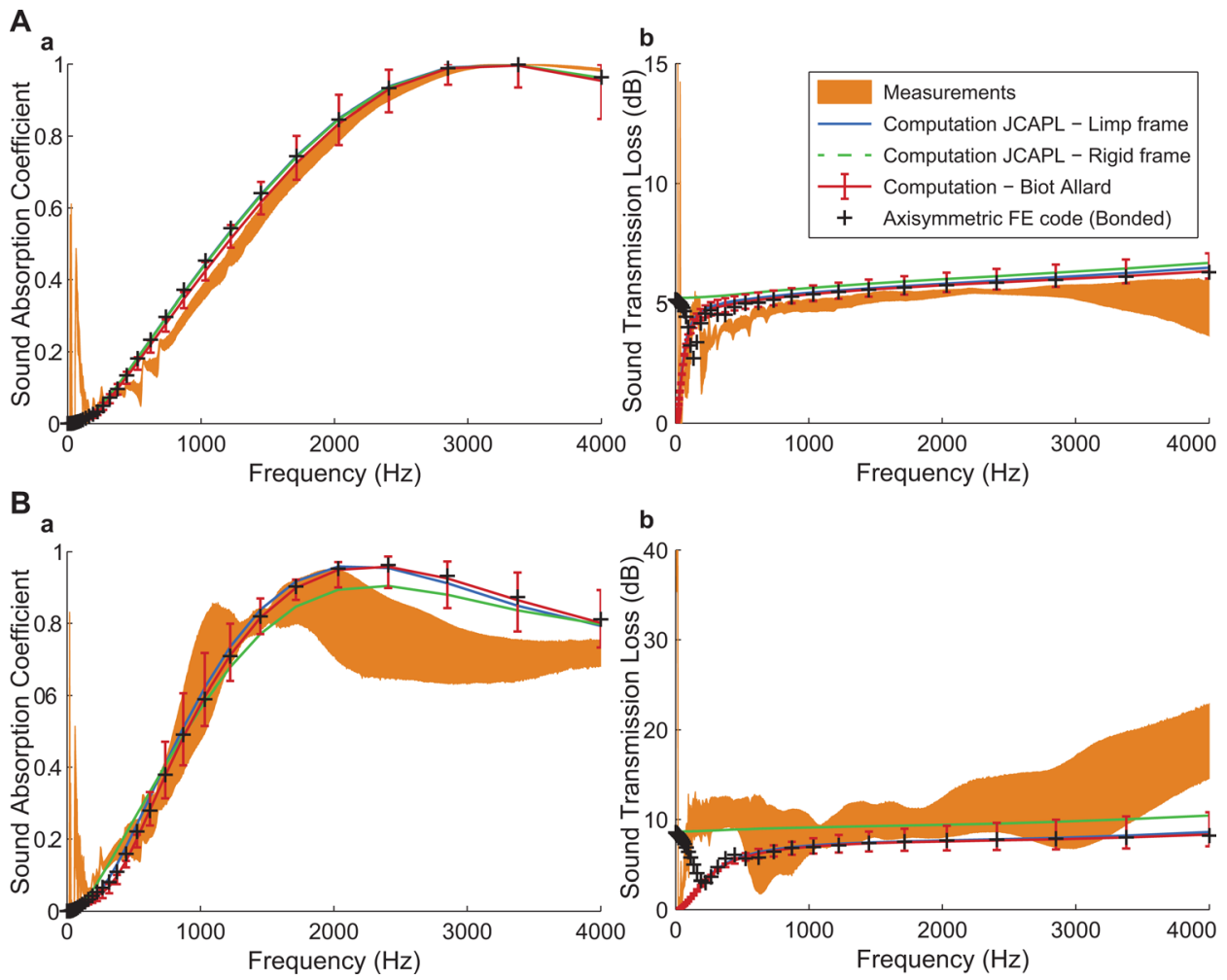

FIG. 6. (Color online) Acoustical properties of two different poroelastic foam samples, $H_{1}(\mathrm{~A})$ and $H_{2}(\mathrm{~B})$. also observed numerically that the dip in the experimental sound transmission loss is properly reproduced by the poroelastic FE formulation when the Young's modulus corresponding to a compression rate $\sim 3 \%$ is used (Fig. 4); the effective Young's modulus of the foam was significantly increased by the mounting conditions in the tube. Modeling this effect by a micro-macro approach will be the topic of a future work. Also, it is worth mentioning that the very large range of measurement dispersion observed for $\mathrm{H}_{2}$ [Fig. 6(B)] may be understood as a direct consequence of its very low Young's modulus (Figs. 4 and 5) originating from the constituting material itself ( $\mathrm{H}_{2}$ displays a higher closure rate of membranes, which tends to rigidify the cellular structure). Understanding these dominant mechanisms as an overall consistent picture strongly supports the interest of this type of calculation. The results of this analysis, therefore, show that the elastic properties derived from the micromechanical model are directly relevant for the determination of the acoustical properties of real foam samples. Using a glued sample between the rear face and hard wall, as an experimental boundary condition in order to reinforce the sensitivity of the elastic constants of the frame to acoustic excitation, will be dealt in a future analysis.

\section{CONCLUDING REMARKS}

Let us have an overall view of the results that were derived from this multi-scale approach with the poroelastic foam samples used in this paper (Fig. 1). For the sake of clarity, let us recall the procedure. The porosity, $\phi$, permeability, $k_{0}$, and ligament length, $L$, are assumed to be known from the measurements. In the multi-scale approach, the extension of the solid film constituting the membranes was implemented at growing rates. The cell size is known from SEM experiments and the closure rate of membranes is adjusted for obtaining the experimental permeability. Macroscopic parameters are then computed from numerical homogenization and compared to the values that were measured at macro-scale in Sec. IV and gathered in Tables I and IV. These latter values serve in a way as bridges between microstructure and acoustical macro-behavior with microphysical and micromechanical foundations. The numerical simulations are generally in good agreement with the standing wave tube measured values. As also shown above, the proposed micromechanical method can provide reasonable estimates of linear elastic properties for poroelastic foams including the significant effects of membranes' closure rate and thickness. The method is based on the use of a simplified cellular morphology with identified local characteristic lengths, a so-called "idealized periodic unit cell." Further systematic investigation on the sensitivity of the results with regard to the choice of particular features of the cellular morphology should be carried out. It is to be noted that accurate values for the Young's modulus and Poisson's ratio of the base material are difficult to obtain because of the variability of the base material itself encountered in the foaming process and the need to implement advanced characterization techniques at this scale. In other words, extending this multiscale method to real life sound insulation optimization problems is not straightforward, but the present methodology should readily be extended.

The scaling of the 3D membrane-based unit cell was obtained through both numerical simulations and macroscopic measurements $\left(\phi, k_{0}\right)$ following the critical-path argument discussed in Refs. 11 and 16. The experimentally measured pore and throat sizes were shown to agree fairly 
well with calculated (Fig. 3) sizes, meaning that the local geometry model can be obtained by measurements of the microstructure itself, which opens a new robust characterization and optimization avenue.

To our knowledge, the present method and procedure for the calculation of mechanical and fluid flow properties from partially open cell membrane-based solid structures are new. Although our micromechanical approach for the linear elastic properties determination is rather classical in the field of numerical homogenization techniques, the same is not true for the generalization of the calculation to all coefficients entering into the macroscopic theory of longwavelength acoustic wave propagation and dissipation through poroelastic media in relation with their microstructures-except for the damping loss factor, $\eta$. One would then have to use the full dynamic equations for the calculation of $\eta$ (and not only the quasi-static ones). Another possible complication is the non-linear behavior that can result from the compression rate of the foam.

Generating precise control over pore morphology 48,49 and mechanical properties ${ }^{50}$ of the base material makes this multi-scale approach particularly promising for various advanced applications. See, in particular, Sec. 3 of Ref. 50, where the principles enabling to tune the mechanical properties of the base material are addressed from the chemistry point of view.

To summarize, we have developed a general methodology in which the morphological dependence of intrinsic parameters in the acoustics of poroelastic foam samples can be considered in a unified framework.

\section{ACKNOWLEDGMENTS}

The authors thank ANRT and Faurecia company for financial support (CIFRE No. 748/2009). Partial support for this work was also provided by Université Paris-Est (mobility grant from the ED SIE) and Université Paris-Est Marnela-Vallée (BQR No. FG 354/2009). We acknowledge R. Combes (LGE, Université Paris-Est) for membrane thickness estimates through SEM imaging, T. Niemeyer for compression testing together with L. Bischoff, A. Duval, and J.-F. Rondeau for helpful input and fruitful discussions (Faurecia), S. Gutierrez for SEM imaging at low magnifications of the solid foam samples (CCM, Université de Sherbrooke), R. Panneton for providing the in-house FE axisymmetric poroelastic formulation and for subsequent stimulating exchanges, F. Chevillotte and L. Jaouen for discussions about data analysis (Matelys), and M. H. Alexandre for English editing assistance.

${ }^{1}$ M. T. Hoang and C. Perrot, "Identifying local characteristic lengths governing sound wave properties in solid foams," J. Appl. Phys. 113, 084905 (2013).

${ }^{2}$ L. Gong, S. Kyriakides, and W.-Y. Jang, "Compressive response of opencell foams. Part I: Morphology and elastic properties,” Int. J. Solids Struct. 42, 1355-1379 (2005).

${ }^{3}$ W.-Y. Jang, S. Kyriakides, and A. M. Kraynik, "On the compressive strength of open-cell metal foams with Kelvin and random cell structures," Int. J. Solids Struct. 47, 2872-2883 (2010).

${ }^{4}$ T. Lévy, "Propagation of waves in a fluid-saturated porous elastic solid," Int. J. Eng. Sci. 17, 1005-1014 (1979).
${ }^{5}$ J. L. Auriault, "Dynamic behavior of a porous medium saturated by a Newtonian fluid," Int. J. Eng. Sci. 18, 775-785 (1980).

${ }^{6} \mathrm{R}$. Burridge and J. B. Keller, "Poroelasticity equations derived from microstructure," J. Acoust. Soc. Am. 70, 1140-1146 (1981).

${ }^{7}$ F. Paun, S. Gasser, and L. Leylekian, "Design of materials for noise reduction in aircraft engines," Aerosp. Sci. Technol. 7, 63-72 (2003).

${ }^{8}$ St. Gasser, "Etude des propriétés acoustiques et mécaniques d'un matériau métallique poreux modèle à base de sphères creuses de nickel (Study of the acoustical and mechanical properties of a model porous metallic material made of hollow nickel spheres)," Ph.D. thesis, Institut National Polytechnique de Grenoble, Grenoble, 2003, pp. 1-306.

${ }^{9}$ S. Gasser, F. Paun, and Y. Bréchet, "Absorptive properties of rigid porous media: Application to face centered cubic sphere packing," J. Acoust. Soc. Am. 117, 2090-2099 (2005).

${ }^{10}$ I. Malinouskaya, V. V. Mourzenko, J.-F. Thovert, and P. M. Adler, "Wave propagation through saturated porous media," Phys. Rev. E 77, 066302 (2008).

${ }^{11}$ M. T. Hoang and C. Perrot, "Solid films and transports in cellular foams," J. Appl. Phys. 112, 054911 (2012).

${ }^{12}$ F. Chevillotte, C. Perrot, and E. Guillon, "A direct link between microstructure and acoustical macro-behavior of real double porosity foams," J. Acoust. Soc. Am. 134, 4681-4690 (2013).

${ }^{13}$ R. M. Sullivan, L. J. Ghosn, and B. A. Lerch, "A general tetrakaidecahedron model for open-celled foams," Int. J. Solids Struct. 45, 1754-1765 (2008).

${ }^{14}$ R. M. Sullivan, L. J. Ghosn, B. A. Lerch, and E. H. Baker, "Elongated tetrakaidecahedron micromechanics model for space shuttle external tank foams," NASA/TP 215137 (2009), pp. 1-23.

${ }^{15}$ P. Thiyagasundaram, B. V. Sankar, and N. K. Arakere, "Elastic properties of open-cell foams with tetrakaidecahedral cells using finite element analysis," AIAA J. 48, 818-828 (2010).

${ }^{16}$ C. Perrot, F. Chevillotte, M. T. Hoang, G. Bonnet, F.-X. Bécot, L. Gautron, and A. Duval, "Microstructure, transport, and acoustic properties of open-cell foam samples: Experiments and three-dimensional numerical simulations," J. Appl. Phys. 111, 014911 (2012).

${ }^{17}$ Y. Salissous and R. Panneton, "Pressure/mass method to measure open porosity of porous solids," J. Appl. Phys. 101, 124913 (2007).

${ }^{18}$ Y. Champoux and J. F. Allard, "Dynamic tortuosity and bulk modulus in air-saturated porous media," J. Appl. Phys. 70, 1975-1979 (1991).

${ }^{19}$ M. R. Stinson and G. A. Daigle, "Electronic system for the measurement of flow resistance," J. Acoust. Soc. Am. 83, 2422-2428 (1988).

${ }^{20}$ D. L. Johnson, J. Koplik, and R. Dashen, "Theory of dynamic permeability and tortuosity in fluid-saturated porous media," J. Fluid Mech. 176, 379-402 (1987).

${ }^{21}$ D. Lafarge, P. Lemarinier, J. F. Allard, and V. Tarnow, "Dynamic compressibility of air in porous structures at audible frequencies," J. Acoust. Soc. Am. 102, 1995-2006 (1997).

${ }^{22}$ J. Rubinstein and S. Torquato, "Diffusion-controlled reactions: Mathematical formulation, variational principles, and rigorous bounds," J. Chem. Phys. 88, 6372-6380 (1988).

23."Homogenization in mechanics of materials," edited by M. Bornert, T. Bretheau, and P. Gilormini (ISTE, London, 2006), pp. 1-448.

${ }^{24}$ J. C. Michel, H. Moulinec, and P. Suquet, "Effective properties of composite materials with periodic microstructure: A computational approach," Comput. Methods Appl. Mech. Eng. 172, 109-143 (1999).

${ }^{25} \mathrm{R}$. Panneton and X. Olny, "Acoustical determination of the parameters governing viscous dissipation in porous media," J. Acoust. Soc. Am. 119, 2027-2040 (2006)

${ }^{26} \mathrm{X}$. Olny and R. Panneton, "Acoustical determination of the parameters governing thermal dissipation in porous media," J. Acoust. Soc. Am. 123, 814-824 (2008).

${ }^{27}$ H. Utsuno, T. Tanaka, T. Fujikawa, and A. F. Seybert, "Transfer function method for measuring characteristic impedance and propagation constant of porous materials," J. Acoust. Soc. Am. 86, 637-643 (1989).

${ }^{28}$ H. X. Zhu, J. F. Knott, and N. J. Mills, "Analysis of the elastic properties of open-cell foams with tetrakaidecahedral cells," J. Mech. Phys. Solids 45, 319-343 (1997).

${ }^{29}$ L. D. Artavia and C. W. Macosko, "Polyurethane flexible foam formation,") in Low Density Cellular Plastics: Physical Basis of Behavior, edited by N. C. Hilyard and A. Cunningham (Chapman and Hall, London, 1994), pp. 22-55.

${ }^{30}$ R. D. Priester and R. B. Turner, "The morphology of flexible polyurethane matrix polymers," in Low Density Cellular Plastics: Physical Basis of Behavior, edited by N. C. Hilyard and A. Cunningham (Chapman and Hall, London, 1994), pp. 78-103.

${ }^{31}$ J. Lecomte-Beckers, "Cours de physique des matériaux: Partie Polymères (Lecture notes on physics of materials)," Université de Liège, 2009, Chap. 8, 
pp. 1-7. Available at http://www.metaux.ulg.ac.be/Fichierpourtelech/polym/ ch\%208.pdf (Last viewed 11/14/2012).

${ }^{32}$ E. Mariez, S. Sahraoui, and J. F. Allard, "Elastic constants of polyurethane foam's skeleton for Biot model," in Proceedings of Internoise96 (1996), pp. 951-954.

${ }^{33}$ C. Langlois, R. Panneton, and N. Atalla, "Polynomial relations for quasistatic mechanical characterization of isotropic poroelastic materials," J. Acoust. Soc. Am. 110, 3032-3040 (2001).

${ }^{34}$ A. Geslain, O. Dazel, J.-P. Groby, S. Sahraoui, and W. Lauriks, "Influence of static compression on mechanical parameters of acoustic foams," J. Acoust. Soc. Am. 130, 818-825 (2011).

${ }^{35}$ T. Pritz, "Transfer function method for investigating the complex modulus of acoustic materials: Spring-like specimens," J. Sound Vib. 72, 317-341 (1980).

${ }^{36}$ O. Danilov, F. Sgard, and X. Olny, "On the limits of an 'in vacuum' model to determine the mechanical parameters of isotropic poroelastic materials," J. Sound Vib. 276, 729-754 (2004).

${ }^{37} \mathrm{~T}$. Iwase, Y. Izumi, and R. Kawabata, "A new measuring method for sound propagation constant by using sound tube without any air spaces back of a test material," in Proceedings of Internoise98, Christchurch, New Zealand (November 16-18, 1998).

${ }^{38}$ Y. Salissou and R. Panneton, "Wideband characterization of the complex wave number and characteristic impedance of sound absorbers," J. Acoust. Soc. Am. 128, 2868-2876 (2010).

${ }^{39}$ B. Brouard, D. Lafarge, and J. F. Allard, "A general method of modelling sound propagation in layered media," J. Sound Vib. 183, 129-142 (1995).

${ }^{40} \mathrm{R}$. Panneton, "Comments on the limp frame equivalent fluid model for porous media," J. Acoust. Soc. Am. 122, EL217-EL222 (2007).

${ }^{41}$ D. Pilon, R. Panneton, and F. Sgard, "Behavioral criterion quantifying the edge-constrained effects on foams in the standing wave tube," J. Acoust. Soc. Am. 114, 1980-1987 (2003).
${ }^{42}$ M. A. Biot, "Theory of propagation of elastic waves in a fluid-saturated porous solid. I. Low frequency range,” J. Acoust. Soc. Am. 28, 168-178 (1956).

${ }^{43} \mathrm{M}$. A. Biot, "Theory of propagation of elastic waves in a fluid-saturated porous solid. II. Higher frequency range," J. Acoust. Soc. Am. 28, 179-191 (1956).

${ }^{44} \mathrm{D}$. L. Johnson, "Recent developments in the acoustic properties of porous media," in Proceedings of the International School of Physics Enrico Fermi, Course XCIII, edited by D. Sette (North Holland, Amsterdam, 1986), pp. 255-290.

${ }^{45}$ J. F. Allard and N. Atalla, "Biot theory of sound propagation in porous materials having an elastic frame," in Propagation of Sound in Porous Media: Modelling Sound Absorbing Materials, 2nd ed. (Wiley, West Sussex, UK, 2009), Chap. 6, pp. 111-135.

${ }^{46} \mathrm{D}$. Pilon, "Influence des conditions aux limites sur les mesures acoustiques au tube à ondes stationnaires (Influence of boundary conditions on the acoustical measurements in a standing wave tube)," M.Sc. thesis, Université de Sherbrooke, Quebec, 2002, pp. 1-260.

${ }^{47}$ D. Pilon, R. Panneton, and F. Sgard, "Convergence of Nth order Biot poroelastic finite elements," in Proceedings of the 9th International Conference on Sound and Vibration, Orlando, FL, 8-11 July 2002.

${ }^{48} \mathrm{~A}$. Testouri, "Highly structured polymer foams from liquid foam templates using millifluidic lab-on-a-chip techniques," Ph.D. thesis, Université Paris Sud, Paris, 2012, pp. 1-167.

${ }^{49}$ A. Testouri, L. R. Arriaga, C. Honorez, M. Ranft, J. Rodrigues, A. van der Net, A. Lecchi, A. Salonen, E. Rio, R.-M. Guillermic, D. Langevin, and W. Drenckhan, "Generation of porous solids with well-controlled morphologies by combining foaming and flow chemistry on a Lab-on-aChip," Colloids Surf. A 413, 17-24 (2012).

${ }^{50}$ A. Duval, M. T. Hoang, V. Marcel, and C. Perrot, "Development of acoustically effective foams: A new micro-macro optimization method," in Polyurethan2012 (Nürtingen, Germany, 2012). 